\title{
Single-cell RNA sequencing reveals the heterogeneity of peripheral blood mononuclear cells in renal cell carcinoma
}

Wei Li

928th Hospital of PLA Joint Logistic Support Force

Kaijian Wang

928th Hospital of PLA Joint Logistic Support Force

Yi Dong

Hainan Hospital of PLA General Hospital

Zhen Deng

900th Hospital of PLA Joint Logistic Support Force

Wei Zhang

81th Hospital, Nanjing University of Chinese Medicine

Hongfeng Shen ( $\square$ shenhongfeng603@126.com )

928th Hospital of PLA Joint Logistic Support Force

\section{Research Article}

Keywords: Single-cell RNA sequencing, heterogeneity, peripheral blood mononuclear cells, renal cell carcinoma

Posted Date: October 25th, 2021

DOI: https://doi.org/10.21203/rs.3.rs-971132/v1

License: (c) (i) This work is licensed under a Creative Commons Attribution 4.0 International License.

Read Full License 


\section{Abstract \\ Background}

Peripheral blood mononuclear cells (PBMCs) are closely related to tumors, and the functions of T cells and $B$ cells are related to tumor occurrence, development, and prognosis. Conventional second-generation sequencing cannot distinguish the characteristics of various peripheral blood mononuclear cell subsets, so it is impossible to study PBMCs accurately. Recently, single-cell sequencing technology has improved, and it provides a tool to study immune cells in circulating blood.

\section{Methods}

PBMCs from patients with renal cell carcinoma (RCC) were sequenced to explore the characteristics of PBMC subsets in patients with renal cell carcinoma, mainly B cells and Treg cells, to study the relationship between renal cell carcinoma and peripheral blood immune cells.

\section{Results}

our PBMC study of RCC patients successfully separated several types of immune cells from the blood. PBMCs can map the tumor to a certain extent.

\section{Conclusions}

We can expand the samples based on the current research, and further research will discover more meaningful information related to cancer.

\section{Background}

Renal cell carcinoma (RCC) is the most common type of renal tumor arising from the proximal renal tubules [. The 5-year survival rate is $44-92 \%$ for RCC patients who are diagnosed when the cancer is localized, and the 5-year survival rate decreases when it has spread to regional lymph nodes or metastasized [2]. Early RCC symptoms are not typical, and they are often overlooked. Therefore, only latestage RCC patients can be diagnosed when the tumor is large and the symptoms are obvious. Peripheral blood mononuclear cells (PBMCs) are composed of lymphocytes (T cells, B cells, NK cells) and monocytes. They have been shown to be involved in the formation and development of many diseases [3]. Using single-cell sequencing technology, previous studies have shown that immune cells in circulating blood are associated with tumors, autoimmune diseases, inflammation, and other diseases $[4,5]$.

Currently, single-cell RNA sequencing (scRNAseq) is an important tool to establish cell lineage and identify tissue composition. Traditional RNA sequencing can only provide the average expression signal 
in the whole tissue without considering cell heterogeneity. However, scRNAseq can also describe subtle differences in cell subsets in complex mixtures such as tissues or blood samples [6, 7]. In this study, PBMCs from renal cancer patients were sequenced to explore the characteristics of mononuclear cell subsets in RCC patients, and to study the relationship between RCC and PBMCs.

\section{Methods}

Ethics statement. The present study was approved by the Ethics Committee at Hainan Hospital. All samples were obtained from patients who were diagnosed with RCC between January 2019 and January 2020 at Hainan Hospital. All study subjects provided written informed consent.

Human samples.Samples were collected from four patients who were initially diagnosed with RCC. Blood was collected from the cubital vein before drug treatment or other antineoplastic therapy. PBMC was isolated from human peripheral blood using Ficoll-Paque density gradient centrifugation. First, whole blood was diluted with the same amount of phosphate buffered saline (PBS), and the diluted blood solution was placed onto the Ficoll-Paque gradient medium (GE Healthcare Bio-Sciences), centrifuged at room temperature at $1000 \times g$ for $20 \mathrm{~min}$, and the PBMCs were carefully collected from the interface layer between plasma and Ficoll solution. The collected PBMCs were washed using PBS, then centrifuged at $500 \times g$ for $20 \mathrm{~min}$, and the cell concentration was adjusted to $1 \times 10^{7} \mathrm{PBMCs} / \mathrm{mL}$. A single cell RNA library was then constructed.

Single-cell cDNA amplification and RNA-sequencing. According to the manufacturer's instructions, PBMCs were labeled with BD Human single Cell Multiplexing Kit(BD Biosciences,Cat No.:633793,San Jose, CA, USA). The cell survival rate for all samples was greater than $80 \%$. The labeled samples were evenly mixed in cold BD sample buffer, and each sample had about 30,000-40,000 cells. Single cells were isolated by single cell capture and gene synthesis using the BD Rhapsody Express single cell analysis system, and the library was constructed using a BD Rhapsody DNA full transcriptome analysis and amplification kit.(BD Biosciences, Cat No.:633781,San Jose, CA,USA). The final library was quantified using a Qubit fluorometer with Qubit dsDNA HS Kit (Thermo Fisher, Cat No.:Q32851,Wilmington, Delaware USA). Libraries were sequenced in the paired-end mode on a NovaSeq 6000 by Novogene Biotech Co., Ltd (Beijing, China).

Processing and analysis of single-cell RNA-seq data. The Seurat Package (version:3.1.2) was used for gene expression data analysis. Cell demultiplexing was realized using the HTODemUX function in the Seurat Package. After single cell identification, cells with mitochondrial readings of more than $30 \%$, fewer than 200 genes, or more than $\mathbf{5 0 0 0}$ genes are excluded from the analysis. Downstream analysis only considered those genes that exist in more than five cells, and standardization, scaling, and dimensionality reduction steps were performed for each subset of PMBC data. Uniform Manifold Approximation and Projection (UMAP) was then used for two-dimensional representation of the data structure. After clustering, the "findmarkers" and "findallmarkers" functions from the Seurat software package were used to search the clustering biomarkers of each group, and the clustering marker genes were determined by 
the expression differences. Tags that identify a single cluster were identified and compared with all other cells.

Identification of single cell subpopulation identification. There are many automatic tools for single cell subgroup identification, and they are mainly divided into two categories, as follows: automatic recognition and semi-supervised. The more common automatic recognition is Singler, which has built-in cell data from humans and mice. The basic principle is to determine the cell type by calculating the correlation between a single cell and the built-in database. The advantage of this tool is that a person does not need to provide their own cell types and corresponding marker genes, but its disadvantage is that it can only recognize cell types that already exist in the database, and it cannot recognize particularly fine cell subsets. The cell subpopulation can also be identified based on traditional classical marker genes, and cell subsets can be identified based on marker genes of known cell types. Generally, subgroup identification is not a single gene, but may require multiple genes. The traditional classical marker gene collection generally uses the following two commonly used databases:

CellMarker(http://biocc.hrbmu.edu.cn/CellMarker/) and Magi Panglaodb

(https://panglaodb.se/index.html). In this study, we used the traditional classical marker gene, combined with automatic identification tool Singler to identify the cell population.

\section{Results}

Single cell RNA-seq data quality. PBMCs were isolated and RNA-Seq libraries were constructed for sequencing on the Illumina sequencing platform. Single-cell data sets usually contain various forms of rough information such as technical noise and batch effect. Processing these signals can improve downstream dimension reduction and clustering, and improve the reliability of data analysis. We obtained 7659 high-quality cells after quality control, based on the information from genes and mitochondrial genes in the samples (Fig. 1A, B). After data normalization, all hypervariable genes in a single cell were screened, and downstream analysis was performed using the hypervariable genes (Fig. 1C).

Visualization and exploration of single-cell sequencing data. In addition to PCA dimensionality reduction, Seurat provides several nonlinear dimensionality reduction techniques for visualization and exploration of single cell sequencing data, to place similar units together in low dimensional space. Uniform Manifold Approximation and Projection (UMAP) is a recently published nonlinear dimensionality reduction technique. Compared with tSNE, UMAP has a faster running time, better consistency, more meaningful cell cluster organization, and better continuity preservation. In this study, PBMCs were clustered and seven major cell types were identified using UMAP, which included T cells, NK cells, monocytes, B cells, $T$ regulatory (Treg) cells, plasmacytoid dendritic cells, and gamma delta T cells (Fig. 2A). Single cell sequencing was also used to annotate each of our PBMCs with the main cell type labels. The results included a heatmap of the group and label scores (Fig. 2B). Each group should exhibit a high score in one label relative to all of the other scores, indicating that the label assignment was unambiguous. The 
results showed that each cell subgroup can be divided into different cell subtypes. For example, $T$ cells can be divided into CD4+ effector memory, CD8+ effector memory, CD8+ naïve, and other subtypes.

B cell and Treg cell clusters in PBMCs.We identified each cell cluster with the largest proportion of common $T$ and $B$ cells, which is consistent with the actual situation (Fig. 3A, F). The UMAP heat map shows that there is a large distance between $B$ cells and other cell clusters. Further analysis showed that the genes CD79, MS4A1, and IGHM had relatively specific expression in B cell clusters (Fig. 3C-E). We screened highly variable genes from B cells and analyzed these genes using GO terms (Fig. 3B). The results showed that they were mainly enriched in B cell activation, antigen receptor-mediated signaling pathways, antigen processing and presentation of peptide antigens through $\mathrm{MHC}$ class I, regulation of lymphocyte activation, interferon gamma-mediated signaling pathways, regulation of leukocyte proliferation, components of the lumen side of the endoplasmic reticulum, and B cell differentiation.

Tregs are an immunosuppressive subset of CD4+ T cells that are characterized by the expression of the major transcription factor forkhead box protein P3 (Foxp3). Tregs can inhibit tumor immunity, thereby hindering the protective immune surveillance of tumors and the effective anti-tumor immune response of tumor hosts. This promotes tumor development and progression. Our analysis of Tregs and UMAP showed that Foxp3, IL32, and GBP5 were highly expressed in Treg cells (Fig. 3H-J). We screened highly variable Tregs genes and analyzed these genes using $\mathrm{GO}$ terms, and they showed high regulating $\mathrm{T}$ cell activation, leukocyte cell adhesion, regulating lymphocyte activation, lymphocyte differentiation, cytokine secretion, lateral plasma membrane, focal adhesion, and cell-matrix adhesion junctions (Fig. 3G).

\section{Discussion}

In this study, we performed single-cell transcriptome analysis on PBMCs from RCC patients, and then focused on $T$ and $B$ cells. There were more B cells than T cells in bone marrow, but fewer B cells in blood and lymph nodes than T cells and fewer $B$ cells than $T$ cells in the chest tube $[8,9]$. B cells begin as immature cells. Once activated, B cells differentiate into plasma cells and secrete antibodies, especially those against free antigens. In this study, PBMCs were isolated from RCC patients, and a single cell RNA library was constructed using a BD single cell platform, and high-throughput sequencing was performed using a novaseq 6000 sequencing platform. Sequencing results were analyzed and cell clusters were identified. Classification of mononuclear cells showed that $T$ cells and $B$ cells accounted for the largest proportion.B cells were identified from cell clusters, and CD79a, MS4AL and IGHM were highly expressed B cell markers in B cells.GO terminology analysis of highly variable genes in B cell clusters showed that the results conformed to the functional expression of $B$ cells.Seurat is an $\mathrm{R}$ language software package for single-cell RNA sequence data that can identify and explain the source of cellular heterogeneity from single-cell transcriptome sequencing data, and integrate various types of single-cell data for data analysis and mining.

Treg cells are a subset of CD4+ T cells that have a significant immunosuppressive effect, which can inhibit the immune response of other cells, and they play an important role in maintaining the immune 
balance and preventing autoimmune diseases and transplantation rejection $[10,11]$. Treg cells can be divided into natural Treg cells and induced Treg cells, but in this study, they were not further classified. In peripheral blood, Treg cells account for $5-10 \%$ of the total number of CD4+ T cells $[12,13]$. Currently, it is believed that natural Tregs are derived from the thymus and that they play an inhibitory role mainly through cell contact mechanism [10]. iTregs are induced by peripheral mature T cells under conditions of persistent stimulation by antigens as well as transforming growth factor (TGF- $\beta$ ) and other cytokines [14].

Foxp3, IL32, and GBP5 are markers of Treg cell subsets. The G0 term analysis in this study mainly included an increase in Treg activation, leukocyte cell adhesion, regulating lymphocyte activation, and lymphocyte differentiation. The GO terms are in agreement with the biological information from Treg cells, which supports the authenticity of our data.

This study had some limitations including a small sample size. However, we only detected the PBMC transcriptome and preliminarily classified PBMC subpopulations. We did not perform an in-depth analysis, especially in combination with clinical and pathological analysis. Single-cell RNA sequencing is an important tool in tumor research, and this project has provided some information for our future research.

\section{Conclusions}

In sum, our PBMC study of RCC patients successfully separated several types of immune cells from the blood. PBMCs can map the tumor to a certain extent. We can expand the samples based on the current research, and further research will discover more meaningful information related to cancer.

\section{Abbreviations}

PBMCs Peripheral blood mononuclear cells

RCC renal cell carcinoma

scRNAseq single-cell RNA sequencing

PBS phosphate buffered saline

UMAP Uniform Manifold Approximation and Projection

\section{Declarations}

\section{Ethics approval and consent to participate}

All experimental protocols have been performed in accordance with the Declaration of Helsinki and approved by the Ethics Committee 928th Hospital. All methods were carried out in accordance with 
relevant guidelines and regulations. The informed consent was obtained from all subjects and/or their legal guardian(s).

\section{Consent for publication}

Not applicable.

\section{Availability of data and materials}

The datasets that were used during the present study are available from the corresponding author on reasonable request.

\section{Competing interests}

The authors declare that they have no competing interests.

\section{Funding}

This article did not receive sponsorship for publication.

\section{Authors' contributions}

H.S. provided study objective. H.S., W.L., and K.W. designed the study. D.Z. and W.Z. provided technical support. W.L., K.W. and Y.D. designed the sequencing experiments. W.L. performed data analyses. W.L., K.W. and Y.D. interpreted the results and composed the manuscript. All authors critically reviewed the manuscript. W.L. and H.S. are responsible for data integrity. All authors read and approved the final manuscript.

\section{Acknowledgements}

Not applicable.

\section{Author details}

${ }^{1}$ Department of Urology, 928th Hospital of PLA Joint Logistic Support Force, Haikou, Hainan, China.

${ }^{2}$ Department of Urology, Hainan Hospital of PLA General Hospital, Sanya, Hainan, China. ${ }^{3}$ Department of Urology, 900th Hospital of PLA Joint Logistic Support Force, Fuzhou, Fujian, China. ${ }^{4}$ Department of Urology, 81th Hospital affiliated to Nanjing University of Chinese Medicine, Nanjing, Jiangsu, China.

\section{References}

1. Muglia VF, Prando A. Renal cell carcinoma: histological classification and correlation with imaging findings. Radiol Bras. [Journal Article; Review]. 2015 2015-05-01;48(3):166-74. 
2. Young JR, Coy H, Kim HJ, Douek M, Lo P, Pantuck AJ, et al. Performance of Relative Enhancement on Multiphasic MRI for the Differentiation of Clear Cell Renal Cell Carcinoma (RCC) From Papillary and Chromophobe RCC Subtypes and Oncocytoma. AJR Am J Roentgenol. [Evaluation Study; Journal Article]. 2017 2017-04-01;208(4):812-9.

3. Van Belleghem JD, Clement F, Merabishvili M, Lavigne R, Vaneechoutte M. Pro- and antiinflammatory responses of peripheral blood mononuclear cells induced by Staphylococcus aureus and Pseudomonas aeruginosa phages. Sci Rep. [Journal Article; Research Support, Non-U.S. Gov't]. 2017 2017-08-14;7(1):8004.

4. Singh M, Al-Eryani G, Carswell S, Ferguson JM, Blackburn J, Barton K, et al. High-throughput targeted long-read single cell sequencing reveals the clonal and transcriptional landscape of lymphocytes. NAT COMMUN. [Journal Article; Research Support, Non-U.S. Gov't]. 2019 2019-07-16;10(1):3120.

5. Kakaradov B, Arsenio J, Widjaja CE, He Z, Aigner S, Metz PJ, et al. Early transcriptional and epigenetic regulation of CD8(+) T cell differentiation revealed by single-cell RNA sequencing. NAT IMMUNOL. [Journal Article; Research Support, Non-U.S. Gov't; Research Support, N.I.H., Extramural]. $20172017-$ 04-01;18(4):422-32.

6. Papalexi E, Satija R. Single-cell RNA sequencing to explore immune cell heterogeneity. NAT REV IMMUNOL. [Journal Article; Research Support, N.I.H., Extramural; Review]. 2018 2018-01$01 ; 18(1): 35-45$.

7. Wu AR, Neff NF, Kalisky T, Dalerba P, Treutlein B, Rothenberg ME, et al. Quantitative assessment of single-cell RNA-sequencing methods. NAT METHODS. [Comparative Study; Journal Article; Research Support, N.I.H., Extramural; Research Support, Non-U.S. Gov't]. 2014 2014-01-01;11(1):41-6.

8. Sage PT, Ron-Harel N, Juneja VR, Sen DR, Maleri S, Sungnak W, et al. Suppression by TFR cells leads to durable and selective inhibition of B cell effector function. NAT IMMUNOL. 2016;17(12):1436-46.

9. Hong S, Zhang Z, Liu H, Tian M, Zhu X, Zhang Z, et al. B Cells Are the Dominant Antigen-Presenting Cells that Activate Naive CD4(+) T Cells upon Immunization with a Virus-Derived Nanoparticle Antigen. IMMUNITY. [Journal Article; Research Support, Non-U.S. Gov't]. 2018 2018-10-16;49(4):695708.

10. Tanaka A, Sakaguchi S. Regulatory T cells in cancer immunotherapy. CELL RES. [Journal Article; Review]. 2017 2017-01-01;27(1):109-18.

11. Tanaka A, Sakaguchi S. Targeting Treg cells in cancer immunotherapy. EUR J IMMUNOL. [Journal Article; Research Support, Non-U.S. Gov't; Review]. 2019 2019-08-01;49(8):1140-6.

12. Shaoqing Y, Yinjian C, Zhiqiang Y, Ruxin Z, Na C, Rongming G. The levels of CD4+CD25+ regulatory $T$ cells in patients with allergic rhinitis. Allergol Select. [Journal Article]. 2018 2018-01-20;2(1):144-50.

13. Goschl L, Scheinecker C, Bonelli M. Treg cells in autoimmunity: from identification to Treg-based therapies. SEMIN IMMUNOPATHOL. [Journal Article; Review]. 2019 2019-05-01;41(3):301-14.

14. Kanamori M, Nakatsukasa H, Okada M, Lu Q, Yoshimura A. Induced Regulatory T Cells: Their Development, Stability, and Applications. TRENDS IMMUNOL. [Journal Article; Review; Research Support, Non-U.S. Gov't]. 2016 2016-11-01;37(11):803-11. 
Figures

A nFeature_RNA nCount_RNA
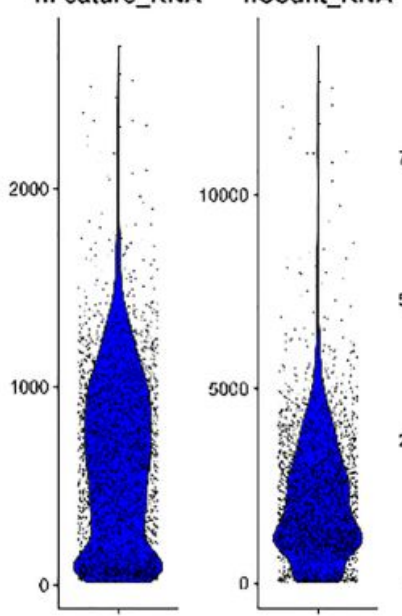

nCount_RNA percent.mt

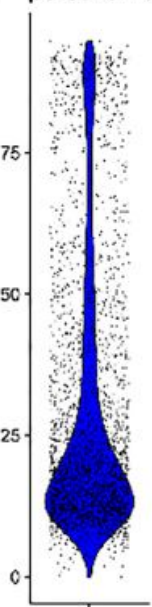

B

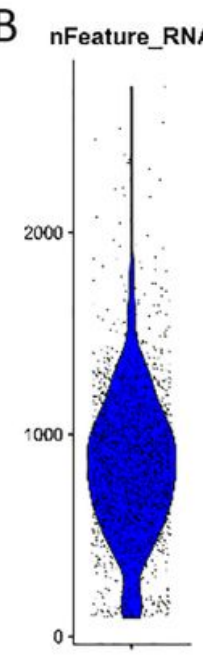

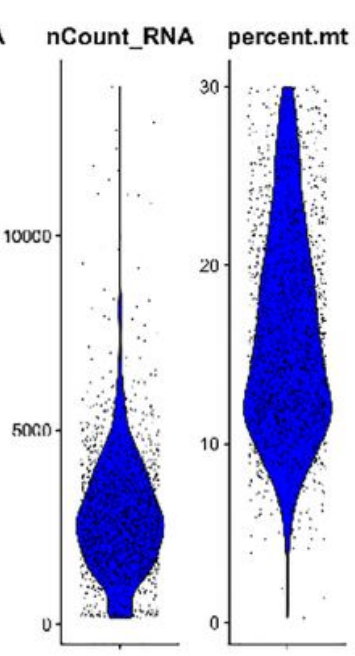

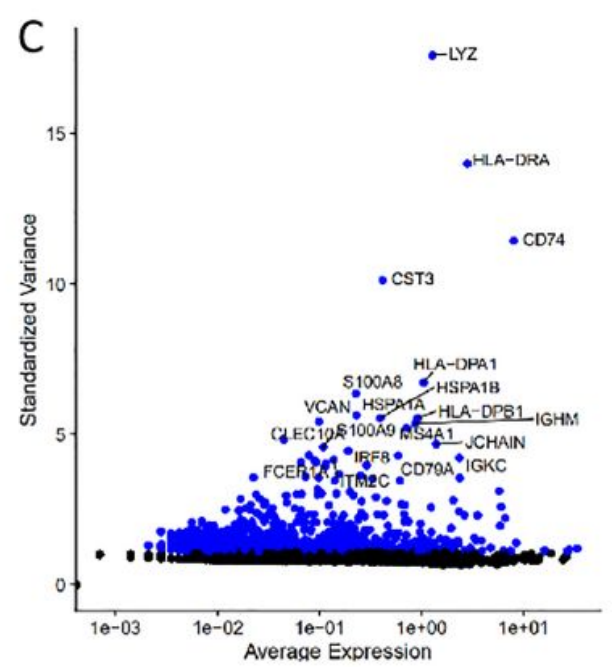

\section{Figure 1}

Single-cell sequencing quality control. A, cell count, gene number, and mitochondrial gene percentage before quality control; B, cell count, gene number, and mitochondrial gene percentage after quality control; $\mathrm{C}$, screening of highly variable genes. 

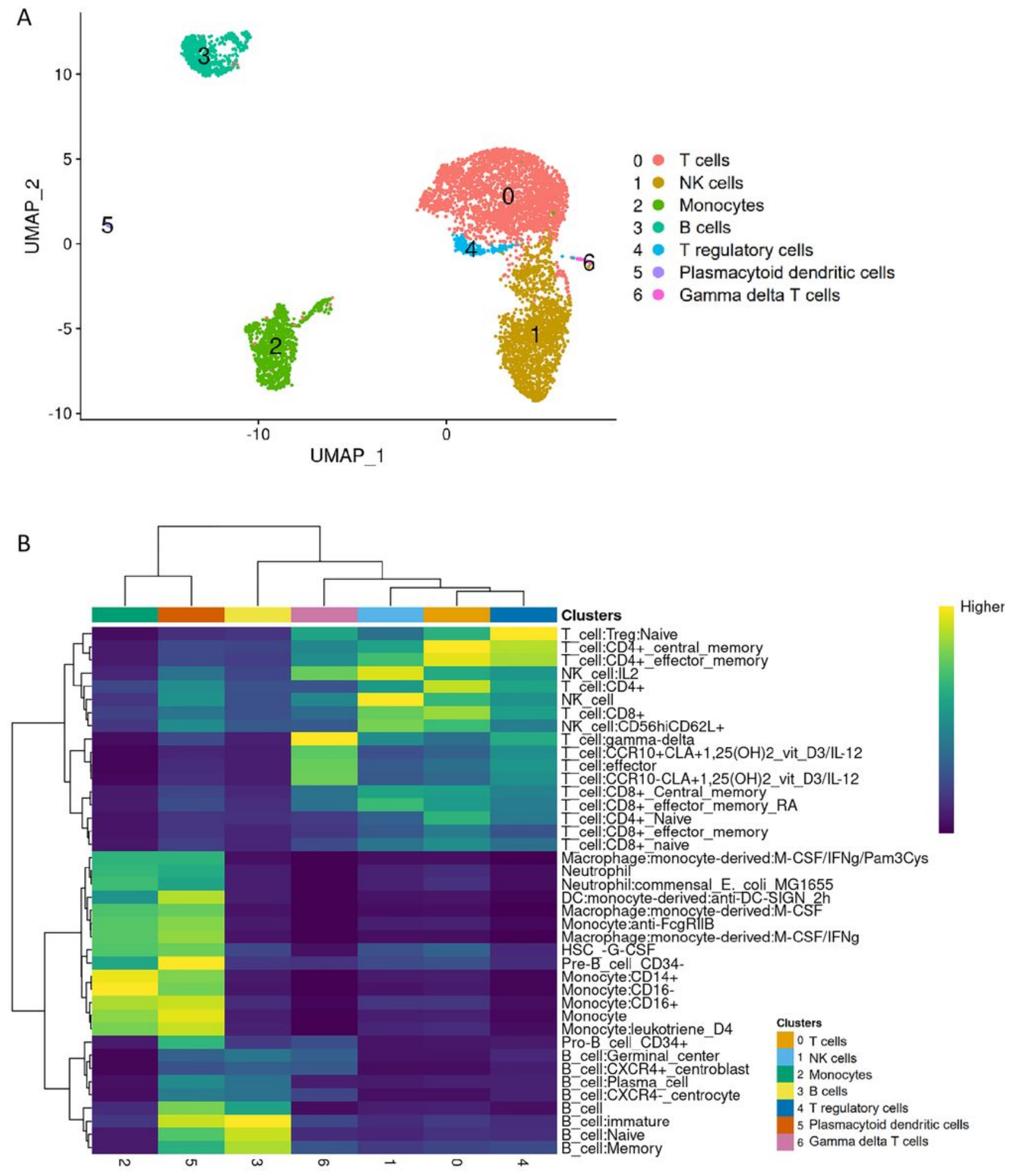

Figure 2

Heatmap of the assignment score for PBMCs. A, PBMC classification and nomenclature; B, heatmap of the assignment score for each cell (column) and label (row), Scores are shown before any fine-tuning within each group. 

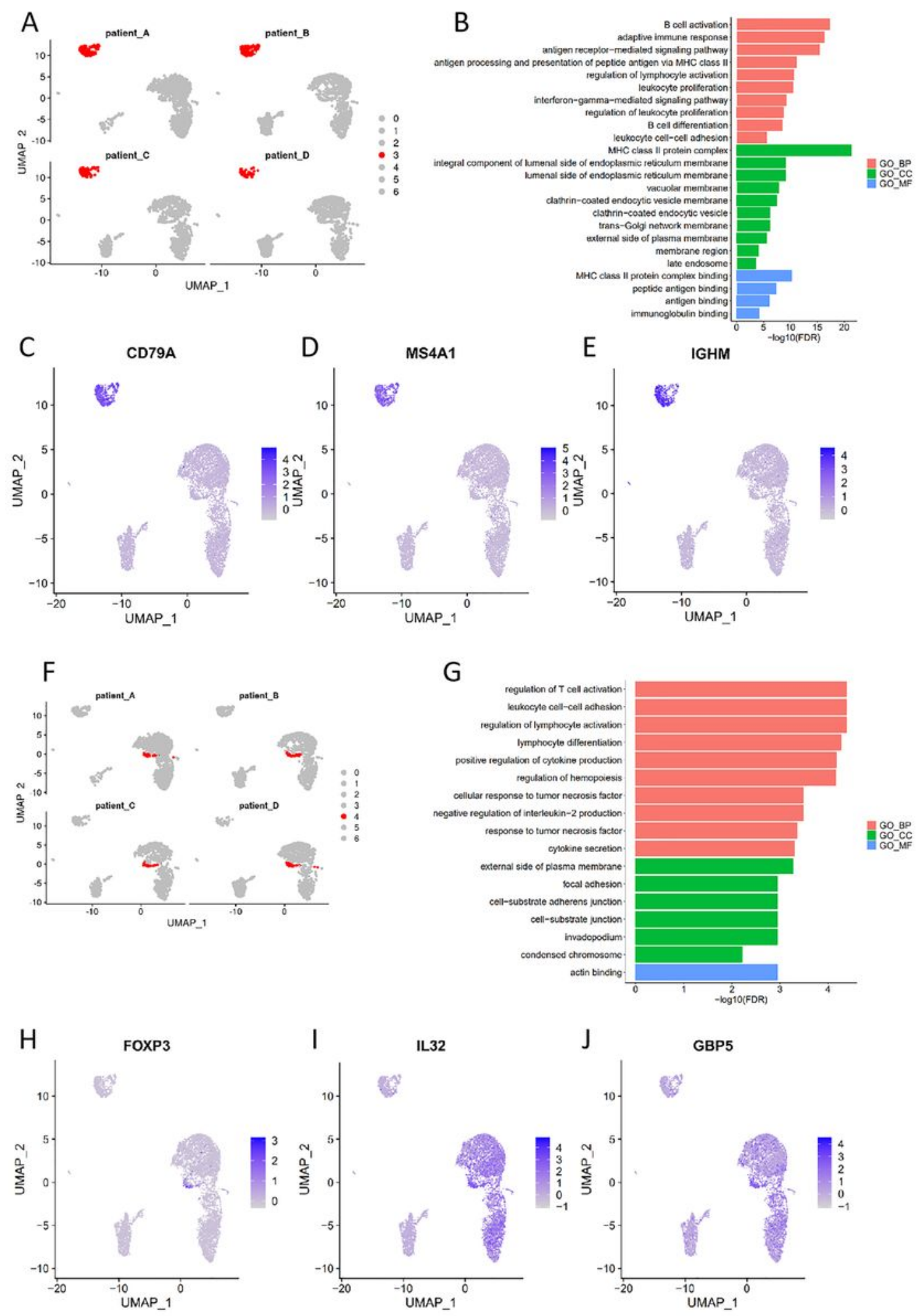

\section{Figure 3}

B cell and Treg cell clusters in PBMCs. A, B cell distribution in four patient populations; B, specific markers of the $\mathrm{GO}$ term; C-E, specific markers of B cells; F, Treg cell distribution in four patient populations; $G$, specific markers of the $\mathrm{GO}$ term; $\mathrm{H}-\mathrm{J}$, specific markers of Treg cells. 